\title{
Gears, Pregears and Related Domains
}

October 6, 2018

Philip R. Brown 1

R. Michael Porter ${ }^{1}$

\begin{abstract}
We study conformal mappings from the unit disk to one-toothed gear-shaped planar domains from the point of view of the Schwarzian derivative. Gear-shaped (or "gearlike") domains fit into a more general category of domains we call "pregears" (images of gears under Möbius transformations), which aid in the study of the conformal mappings for gears and which we also describe in detail. Such domains being bounded by arcs of circles, the Schwarzian derivative of the Riemann mapping is known to be a rational function of a specific form. One accessory parameter of these mappings is naturally related to the conformal modulus of the gear (or pregear) and we prove several qualitative results relating it to the principal remaining accessory parameter. The corresponding region of univalence (parameters for which the rational function is the Schwarzian derivative of a conformal mapping) is determined precisely.
\end{abstract}

Keywords: conformal mapping, accessory parameter, Schwarzian derivative, gearlike domain, conformal modulus, topological quadrilateral.

AMS Subject Classification: Primary 30C30; Secondary 30C20, 33 E05.

\section{Introduction}

A special case of a circular quadrilateral is a gear domain with one tooth: a starlike open set in the complex plane bounded by arcs of two circles centered at the origin and segments of two lines passing through the origin. A related family of domains which we call pregear domains, are those which are Möbius transformations of gear domains.

\footnotetext{
${ }^{1}$ Partially supported by CONACyT grant 166183
} 
In [8], the Riemann mapping of the unit disk onto a gear domain, fixing the origin, is expressed as the solution of a first order linear differential equation (see (2) below) which is derived by making use of the starlike property in the unit disk and the boundary behavior of the mapping.

An alternative approach, which we follow here, follows the more general construction of conformal maps onto circular polygons, expressing the mapping as a solution of the equation which prescribes its Schwarzian derivative, as expounded in [2, 3. 5], [7, p. 70], [9, 10], [12, p. 198], and [15] for circular quadrilaterals. In the present case of gear domains with one tooth, after normalizing the location of the prevertices, the Schwarzian derivative $R_{t, \lambda}(z)$ (see (15)-(16) below) contains the two unknown parameters $t$ (which determines the prevertices) and $\lambda$ (an auxiliary parameter), which in turn determine via the conformal mapping the two natural geometric quantities which specify the gear domain (Figure 1); namely, the ratio of the radii of the outermost and the innermost circle, which we call the gear ratio $\beta$, and half the angle between the rays that form the straight boundary segments, which we call the gear angle $\gamma$.

We describe one-tooth gear domains, summarize the relevant theory from [8], and work out the formula for $R_{t, \lambda}(z)$ in Section 2. The functional relationship between the parameters $t$ and $\lambda$ and the corresponding gear domain is analyzed Section 3, where for convenience we work with the conformal module $M(t)$ of the gear domain. We prove that when $t$ is fixed, for each $\gamma$ there is at most one value of $\lambda$ for which a solution of (18) is a gear. We also prove that when $\gamma$ is fixed, $\beta$ is a monotonic function of $M(t)$ in the full range $0<t<\pi / 2$. On the other hand, when $\beta$ is fixed, $M(t) \rightarrow 0$ as $\gamma \rightarrow 0$ or $\gamma \rightarrow \pi$, which leads to our conjecture that there are exactly two gears (corresponding to two different values of $\gamma$ ) with module $M(t)$, provided $t$ is below a threshold value $t_{\beta}$; for $t=t_{\beta}$ there is only one gear with module $M(t)$ and when $t$ is above the threshold there are no gears at all with this module.

A basic property of the Schwarzian derivative is its invariance under Möbius transformations. Consequently, the solutions $f$ obtained by solving (5) or (18) with the classical normalizations $f(0)=0$ and $f^{\prime}(0)=1$ are, in general, Möbius transforms of one-tooth gear domains. In Section 4 we give a full geometric description of these "pregear" domains and regard them to be of independent interest from the point of view of conformal mapping. The 
family of pregear domains is seen to be bounded by "degenerate pregear domains", which are Möbius transformations of certain unbounded rectilinear quadrilaterals. From this observation we are able to determine precisely the boundary of the region in the $(t, \lambda)$-plane for which $R_{t, \lambda}$ is the Schwarzian derivative of a univalent function (i.e., a conformal mapping to a gear) .

\section{Schwarzian derivative and accessory parameters}

\section{$2.1 \quad$ Gear domains}

In this study a gearlike domain (or gear domain) is a starlike open set $G$ in the complex plane $\mathbb{C}$ bounded by arcs of circles centered at the origin and segments of lines passing through the origin. Occasionally for reasons of normalization of mappings we may use the same term for a translate of a gear domain, in which case we will clarify that the "gear center" may not be the origin. The fundamental study of gearlike domains was initiated in [8] and further results have appeared in [1, 4, 13, 14.

We will consider in particular bounded gearlike domains $G$ with a single "tooth" as in Figure1. The interior angles are defined by $\pi \alpha_{i}$ for $i=1,2,3,4$, where $\alpha_{1}=\alpha_{4}=1 / 2$ and $\alpha_{2}=\alpha_{3}=3 / 2$. We will assume that $G$ is symmetric in the real axis, and that the corresponding prevertices of the conformal mapping are of the form

$$
z_{1}=e^{i t_{1}}, z_{2}=e^{i t_{2}}, z_{3}=e^{-i t_{2}}, z_{4}=e^{-i t_{1}},
$$

$0<t_{1}<t_{2}<\pi$, as can always be obtained by a preliminary transformation of $\mathbb{D}$. The straight edges of $G$ will be be referred to as the tooth edges, which when prolonged meet at the gear center at an angle $2 \gamma$, where $\gamma$ will be termed the gear angle. The two edges of $\partial G$ which are not tooth edges are arcs of circles centered at the gear center: the $A$-arc ending at angles of $3 \pi / 2$ and the $B$-arc ending at angles of $\pi / 2$. The quotient $\beta$ of the radius of the B-arc to that of the A-arc is the gear ratio of $G$. We write $G_{\beta, \gamma}$ for

the standard gear domain with gear parameters $\beta, \gamma$ which is centered at the origin and has A-arc of radius 1. 


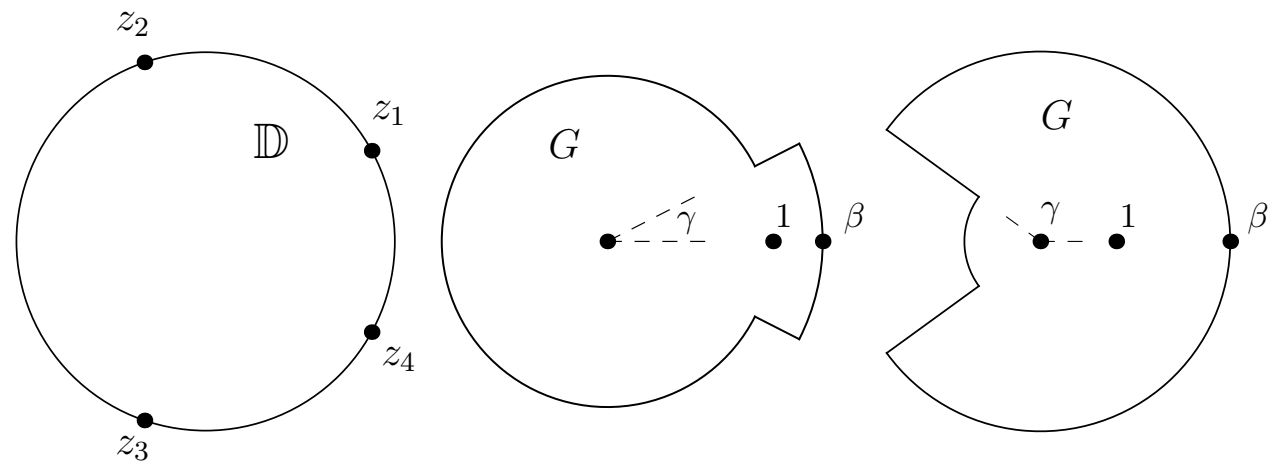

Figure 1: Gear parameters.

Most of the research which has been done on gearlike domains is based on results in [8]. We state here the following particular case.

Proposition 2.1 A necessary and sufficient condition for $f(z)$ to be a univalent mapping of the unit disk onto a one-tooth gear domain, satisfying the normalizations $f(0)=0$ and

$$
f^{\prime \prime}(0)=2 f^{\prime}(0)\left(\cos t_{2}-\cos t_{1}\right)
$$

where the prevertices $e^{ \pm i t_{1}}$ map to the vertices with interior angles $\pi / 2$ and the prevertices $e^{ \pm i t_{2}}$ map to the vertices with interior angles $3 \pi / 2$, is that

$$
f^{\prime}(z)=\frac{1}{z}\left(\frac{z^{2}-2 z \cos t_{1}+1}{z^{2}-2 z \cos t_{2}+1}\right)^{1 / 2} f(z) .
$$

Furthermore, the gear ratio $\beta$ and the gear angle $\gamma$ are determined by the following two integral formulas:

$$
\log \beta=\int_{t_{1}}^{t_{2}} \sqrt{\frac{\cos \theta-\cos t_{2}}{\cos t_{1}-\cos \theta}} d \theta, \quad \gamma=\int_{0}^{t_{1}} \sqrt{\frac{\cos \theta-\cos t_{2}}{\cos \theta-\cos t_{1}}} d \theta .
$$

Proof. The necessity of (2) is a consequence of [8, Theorem 2]. Goodman also derived the formulas for $\beta$ and $\gamma$ and gave formulas for the coefficients $\left\{b_{n}\right\}_{n \geq 2}$ of the Maclaurin series of $f(z)$ in terms of the coefficient $b_{1}$. In particular, he obtained (11). The sufficiency of (2) is a consequence of the geometry and symmetry of a one-tooth gear domain: divide the unit circle $\partial \mathbb{D}$ into four arcs separated by the points $z_{1}, z_{2}, z_{3}, z_{4}$. It follows from (2) as 
in the proofs of [즈, Lemmas 1 and 2] that as $\theta$ increases, $|f|$ is constant and $\arg f$ is increasing for $-t_{1}<\theta<t_{1}$ and $t_{2}<\theta<2 \pi-t_{2}$; similarly arg $f$ is constant and $|f|$ is decreasing for $t_{1}<\theta<t_{2}$, while $\arg f$ is constant and $|f|$ is increasing for $-t_{2}<\theta<-t_{1}$. As a result of the singularities in (2), the interior angles of $f(\partial \mathbb{D})$ at $f\left(e^{ \pm i t_{1}}\right)$ must be $\pi / 2$ and the interior angles at $f\left(e^{ \pm i t_{2}}\right)$ must be $3 \pi / 2$. Now if $f$ were not univalent, then an examination of a few possibilities for which the properties above are satisfied would show that the winding number of $f(\partial \mathbb{D})$ about the origin must be greater than 1 , which means that $f$ could not have a simple zero at the origin. However, this contradicts the property $\lim _{z \rightarrow 0} z f^{\prime}(z) / f(z)=1$.

While it is straightforward to solve (2) numerically, from these relations it is difficult to find the values of $t_{1}, t_{2}$ corresponding to a pair $\beta, \gamma$.

\subsection{The Schwarzian derivative of a gear mapping}

Although in principle the operator $z f^{\prime} / f$ considered by Goodman is simpler than the Schwarzian derivative $\mathcal{S}_{f}=\left(f^{\prime \prime} / f^{\prime}\right)^{\prime}-(1 / 2)\left(f^{\prime \prime} / f^{\prime}\right)^{2}$, we will base our study of gear domains on the latter to take advantage of the rich theory which has developed around it, as referred to in the introduction.

We require some general facts about conformal mappings of circle polygon domains. Let $\mathbb{D}=\{\mid z<1\}$ denote the unit disk. For a general circle polygon domain $D$ with interior angles $\pi \alpha_{k}$ at the vertices $w_{k}$, write $a_{k}=\left(1-\alpha_{k}^{2}\right) / 2$. Let $f: \mathbb{D} \rightarrow D$ be a conformal mapping. Then the Schwarzian derivative $\mathcal{S}_{f}$ is a rational function of the form

$$
\mathcal{S}_{f}(z)=z^{-2} \sum_{k=1}^{n}\left(\frac{a_{k} z_{k} z}{\left(z-z_{k}\right)^{2}}+i r_{k} \frac{z+z_{k}}{z-z_{k}}\right),
$$

where $z_{k}=f^{-1}\left(w_{k}\right) \in \partial \mathbb{D}(1 \leq k \leq n)$ are the prevertices of $D$ with respect to the mapping $f$, and $r_{k} \in \mathbb{R}$ are additional accessory parameters which satisfy the relations

$$
\sum_{k=1}^{n} r_{k}=0, \quad \sum_{k=1}^{n} z_{k}\left(a_{k}+2 i r_{k}\right)=0,
$$

These relations assure that $f$ sends the boundary $\partial \mathbb{D}$ to a union of circular arcs, and further imply that the singularity of (3) at the origin is removable. See [15] for a proof. For one-tooth gear domains this specializes as follows. 
Theorem 2.2 Let $G$ be a one-tooth gear domain and let $f: \mathbb{D} \rightarrow G$ be a conformal mapping. Suppose that $f$ is symmetric in the real axis. Then there are unique values $t_{1}, t_{2}, \lambda\left(0<t_{1}<t_{2}<\pi, \lambda \in \mathbb{R}\right)$ such that Schwarzian derivative $\mathcal{S}_{f}$ of $f$ can be expressed as

$$
S_{f}=R_{t_{1}, t_{2}, \lambda}
$$

where

$$
\frac{1}{2} R_{t_{1}, t_{2}, \lambda}(z)=\psi_{0,\left(t_{1}, t_{2}\right)}(z)-\lambda \psi_{1,\left(t_{1}, t_{2}\right)}(z)
$$

with

$$
\psi_{1,\left(t_{1}, t_{2}\right)}(z)=\frac{4\left(\cos t_{2}-\cos t_{1}\right)}{\left(z^{2}-\left(2 \cos t_{1}\right) z+1\right)\left(z^{2}-\left(2 \cos t_{2}\right) z+1\right)}
$$

and

$$
\begin{aligned}
\psi_{0,\left(t_{1}, t_{2}\right)}(z) & =\frac{c_{40} z^{4}+c_{30} z^{3}+c_{20} z^{2}+c_{10} z+c_{00}}{\left(z^{2}-\left(2 \cos t_{1}\right) z+1\right)^{2}\left(z^{2}-\left(2 \cos t_{2}\right) z+1\right)^{2}}, \\
c_{00} & =c_{40}=\frac{3 \cos 2 t_{1}-5 \cos 2 t_{2}+2}{8}, \\
c_{10} & =c_{30}=3 \sin ^{2} t_{1} \cos t_{2}-5 \cos t_{1} \sin ^{2} t_{2}, \\
c_{20} & =\frac{\left(\cos 2 t_{1}\right)\left(11-2 \cos 2 t_{2}\right)-13 \cos 2 t_{2}+4}{4} .
\end{aligned}
$$

Proof. Via the assumed symmetry $f(z)=\overline{f(\bar{z})}$ we have $\mathcal{S}_{f}(z)=\overline{\mathcal{S}_{f}(\bar{z})}$. From this and the general form (3i) with $n=4$ it follows (using $z_{1}=\exp \left(i t_{1}\right)=\overline{z_{4}}$, $\left.z_{2}=\exp \left(i t_{2}\right)=\overline{z_{3}}\right)$ that $r_{1}=-r_{4}, r_{2}=-r_{3}$, so with the first relation of (4) the general formula reduces to

$$
\begin{aligned}
z^{2} \mathcal{S}_{f}(z)= & \frac{3}{8}\left(\frac{z z_{1}}{\left(z-z_{1}\right)^{2}}+\frac{z \overline{z_{1}}}{\left(z-\overline{z_{1}}\right)^{2}}\right)-\frac{5}{8}\left(\frac{z z_{2}}{\left(z-z_{2}\right)^{2}}+\frac{z \overline{z_{2}}}{\left(z-\overline{z_{2}}\right)^{2}}\right) \\
& +i r_{1}\left(\frac{z+z_{1}}{z-z_{1}}-\frac{z+\overline{z_{1}}}{z-\overline{z_{1}}}\right)+i r_{2}\left(\frac{z+z_{2}}{z-z_{2}}-\frac{z+\overline{z_{2}}}{z-\overline{z_{2}}}\right)
\end{aligned}
$$

We introduce a new real parameter $\lambda$, determined by

$$
r_{1}=\frac{\lambda+(3 / 8) \cos t_{1}}{2 \sin t_{1}}, \quad r_{2}=\frac{\lambda-(5 / 8) \cos t_{1}}{2 \sin t_{2}},
$$

and the second relation of (4). After substituting exp $\pm i t_{1}$ and $\exp \pm i t_{2}$ for the prevertices of $f$, the result follows by algebraic manipulation (see [15, 
Appendix] for further details). Clearly $t_{1}, t_{2}$ are determined by the given prevertices of $f$, and the uniqueness of $\lambda$ follows from the explicit formula.

Symmetrization of prevertices. We are interested in the combinations of accessory parameters $\left(t_{1}, t_{2}, \lambda\right)$ for which $f$ is a gear mapping. We have the flexibilty to relocate the prevertices $t_{1}$ and $t_{2}$, as we explain in the following paragraphs.

The pullback of a gear mapping $f$ via the self-mapping

$$
T_{q}(z)=\frac{z-q}{-q z+1} .
$$

of $\mathbb{D}$ is by definition the Schwarzian derivative of the function $f \circ T_{q}$, also defined in $\mathbb{D}$. Since the composition is also a gear mapping, by Theorem 2.2 and the Chain Rule we obtain the form of the pullback of $\mathcal{S}_{f}$,

$$
R_{t_{1}, t_{2}, \lambda}(z)=R_{t_{1}^{*}, t_{2}^{*}, \lambda^{*}}\left(z^{*}\right) T_{q}^{\prime}(z)^{2}
$$

for some $\lambda^{*} \in \mathbb{R}$, where $\mathcal{S}_{f}=R_{t_{1}^{*}, t_{2}^{*}, \lambda^{*}}\left(z^{*}\right), e^{i t_{1}^{*}}=T_{q}\left(e^{i t_{1}}\right), e^{i t_{2}^{*}}=T_{q}\left(e^{i t_{2}}\right), z^{*}=$ $T_{q}(z)$. It is not difficult to see that the " $\psi_{0}$ " and " $\psi_{1}$ " parts of the Schwarzian derivative pull back independently because of the differing degrees of the polynomials in their denominators:

$$
\begin{aligned}
& \psi_{0 ; t_{1}, t_{2}}(z)=\psi_{0 ; t_{1}^{*}, t_{2}^{*}}\left(z^{*}\right) T_{q}^{\prime}(z)^{2}, \\
& \psi_{1 ; t_{1}, t_{2}}(z)=\psi_{1 ; t_{1}^{*}, t_{2}^{*}}\left(z^{*}\right) T_{q}^{\prime}(z)^{2} .
\end{aligned}
$$

In fact, the following holds.

Proposition 2.3 The pullback of the Schwarzian derivative $\mathcal{S}_{f}$ of a gear mapping respects the auxiliary parameters in the sense that $\lambda^{*}=\lambda$ in (11).

Proof. This is an explicit but tedious calculation making use of the elementary formula

$$
T_{q}(a)-T_{q}(b)=T_{q}^{\prime}(a)^{1 / 2} T_{q}^{\prime}(b)^{1 / 2}(a-b)=\frac{\left(1-q^{2}\right)(a-b)}{(1-q a)(1-q b)}
$$

which can be derived immediately from the the formula for the derivative $T_{q}^{\prime}(z)=\left(1-q^{2}\right) /(-q z+1)^{2}$ (and choosing say $\sqrt{1-q^{2}} /(-q z+1)$ as the indicated square root). 
The following result shows that by means of an appropriate $T_{q}$ it is possible to make the prevertices symmetric in the imaginary axis. This will be useful for our considerations of modules in Section 3 .

Proposition 2.4 Let $0<t_{1}<t_{2}<\pi, z_{1}=e^{i t_{1}}, z_{2}=e^{i t_{2}}$. Define

$$
q=\frac{z_{1}+z_{2}-2 \sqrt{\operatorname{Im} z_{1} \operatorname{Im} z_{2}} \sqrt{z_{1} z_{2}}}{1+z_{1} z_{2}}
$$

where $\sqrt{z_{1} z_{2}}=e^{i\left(t_{1}+t_{2}\right) / 2}$. Then there is a unique $0<t<\pi / 2$ such that

$$
T_{q}\left(z_{1}\right)=e^{i t}, \quad T_{q}\left(z_{2}\right)=e^{i(\pi-t)} .
$$

Proof. First we note that $\bar{q}=q$ because of the relations $z_{1} \overline{z_{1}}=z_{2} \overline{z_{2}}=1$. It follows from the definition (10) that $\left|T_{q}\left(z_{1}\right)\right|=\left|T_{q}\left(z_{2}\right)\right|=1$, and then we calculate that

$$
\begin{aligned}
& T_{q}\left(z_{1}\right)=\frac{z_{1}-z_{2}+2 \sqrt{\operatorname{Im} z_{1} \operatorname{Im} z_{2}} \sqrt{z_{1} z_{2}}}{1-z_{1} z_{2}}, \\
& T_{q}\left(z_{2}\right)=\frac{-z_{2}-z_{1}+2 \sqrt{\operatorname{Im} z_{1} \operatorname{Im} z_{2}} \sqrt{z_{1} z_{2}}}{1-z_{1} z_{2}} .
\end{aligned}
$$

Using the same reasoning we used to show $\bar{q}=q$ it follows that $T_{q}\left(z_{2}\right)=$ $-\overline{T_{q}\left(z_{1}\right)}$, so we may take $t=\arg T_{q}\left(z_{1}\right)$.

Since $T_{q}$ fixes \pm 1 and thus conserves the order of $1, e^{i t_{1}}, e^{i t_{2}},-1$ along $\partial \mathbb{D}$, it follows that $T_{1}^{\prime}(0)>0$, which implies that $|q|<1$.

By Proposition 2.3 we can always express $R_{t_{1}, t_{2}, \lambda}$ as

$$
R_{t_{1}, t_{2}, \lambda}=\left(R_{t, \pi / 2-t, \lambda} \circ T_{q}\right)\left(T_{q}^{\prime}\right)^{2} .
$$

for a parameter $t$ uniquely determined by $t_{1}, t_{2}$ according to Proposition 2.4 . We thus reduce the study of gear mappings to the special case

$$
R_{t, \lambda}:=R_{t, \pi / 2-t, \lambda}
$$

Explicitly, we have a much simpler Schwarzian derivative

$$
\frac{1}{2} R_{t, \lambda}(z)=\psi_{0, t}(z)-\lambda \psi_{1, t}(z)
$$


where

$$
\begin{aligned}
& \psi_{0, t}(z)=\frac{\left(\sin ^{2} t\right)\left(z^{4}-(16 \cos t) z^{3}+(4+2 \cos 2 t) z^{2}-(16 \cos t) z+1\right)}{2\left(z^{4}-(2 \cos 2 t) z^{2}+1\right)^{2}}, \\
& \psi_{1, t}(z)=\frac{-8 \cos t}{z^{4}-(2 \cos 2 t) z^{2}+1} .
\end{aligned}
$$

We make the precautionary observation that even though the prevertices are now symmetric in both coordinate axes, the Schwarzian derivative $R_{t, \lambda}$ is not symmetric in the imaginary axis.

\section{Conformal modules of gear domains}

We discuss some of the relations among $t, \lambda, \beta, \gamma$. First we will treat $t$ as fixed, for the following reason. The conformal module $M\left(G_{\beta, \gamma}\right)>0$ of any gear (or pregear) with prevertices $\pm e^{ \pm i t}$ is by definition the conformal module of the unique conformally equivalent rectangle $(0,1,1+\tau, \tau)$ with imaginary $\tau$; i.e., $\tau=i M(t)$. Thus we can write $M(t)=M\left(G_{\beta, \gamma}\right)$. Note that

$$
\lim _{t \rightarrow 0} M(t)=0 ; \quad \lim _{t \rightarrow \pi / 2} M(t)=\infty
$$

Definition 3.1 We will say that the rational function $R_{t, \lambda}$ is gearlike if there is a solution $f$ of

$$
\mathcal{S}_{f}=R_{t, \lambda}
$$

that is a univalent mapping onto a gear.

Lemma 3.2 Fixt. Then for each $\gamma$ there is at most one value of $\lambda$ for which $R_{t, \lambda}$ is gearlike.

Proof. Different values of $\lambda$ for which $R_{t, \lambda}$ is gearlike would correspond to different values of $\beta$. If two gears have the same gear angle $\gamma$ but different values of $\beta$, one must be contained within the other. By the well-known monotonicity of conformal modules of topological quadrilaterals [11] it is not possible for these gears to have the same $M(t)$.

Proposition 3.3 (i) Let $0<\gamma<\pi$. Then $M\left(G_{\beta, \gamma}\right) \rightarrow \infty$ as $\beta \rightarrow 1$, while $M\left(G_{\beta, \gamma}\right) \rightarrow 0$ as $\beta \rightarrow \infty$. (ii) Let $\beta>1$. Then $M\left(G_{\beta, \gamma}\right) \rightarrow 0$ as $\gamma \rightarrow 0$ or $\gamma \rightarrow \pi$. 
Proof. (i) The Euclidean separation of the vertical sides of the topological quadrilateral $G_{\beta, \gamma}$ tends to 0 as $\beta \rightarrow 1$ while the horizontal sides are bounded away from one another, hence the conformal module tends to $\infty$ ([11, Lemma 4.1]). Similarly, as $\beta \rightarrow \infty$, one may rescale the quadrilateral to see that the separation of the horizontal edges tends to 0 , so the conformal module tends to 0 .

(ii) This limit is harder to see because although the horizontal edges in $G_{\beta, \gamma}$ become arbitrarily close, the rightmost vertical edge also degenerates (the Riemann map with $0 \rightarrow 0$ takes all four vertices close to 1 ). We can decompose the gear as $G_{\beta, \gamma}=\mathbb{D} \cup\left\{e^{i \theta}:|\theta|<\gamma\right\} \cup D_{1}$ where $D_{1}$ is the tooth. (Here $\mathbb{D}$ is not a quadrilateral, just a bigon attached to a vertical side of $D_{1}$ but it can be thought of as a limiting case of a quadrilateral.) By monotonicity of conformal modulus,

$$
\frac{1}{M\left(G_{\beta, \gamma}\right)} \geq \frac{1}{M\left(D_{1}\right)}
$$

Since $M\left(D_{1}\right) \rightarrow 0$ as $\gamma \rightarrow 0$, also $M\left(G_{\beta, \gamma}\right) \rightarrow 0$.

Now let $\gamma$ tend to $\pi$. The arc of $\partial \mathbb{D}$ from $\gamma$ to $2 \pi-\gamma$ is a vertical edge which is very small, since there are curves of length less than $2(\pi-\gamma)$ interior to $G_{\beta, \gamma}$ joining the vertical edges (which are the tooth edges, having length $\beta-1)$ to each other. On the other hand, the Euclidean area of $G_{\beta, \gamma}$ is more than $\pi$, the area of $\mathbb{D}$, independently of $\gamma$. By [11, sec. 4.3],

$$
M\left(G_{\beta, \gamma}\right)<\frac{(2(\pi-\gamma))^{2}}{\pi} \rightarrow 0
$$

as $\gamma \rightarrow \pi$.

As in Lemma 3.2, the function $\beta \mapsto M\left(G_{\beta, \gamma}\right)$ is monotone since the gear grows with $\beta$. By Proposition 3.3 we have the following.

Proposition 3.4 Let $\gamma \in(0, \pi)$. Then for any $t \in(0, \pi / 2)$ there is a unique $\beta>1$ such that the gear $G_{\beta, \gamma}$ has conformal module $M(t)$.

Given $\beta$, the existence of a value $\gamma$ for which $M(G(\beta, \gamma)))$ is maximal follows likewise from Proposition 3.3. The affirmation that the conformal module is monotone for $\gamma$ above and below this value is so far confirmed only by numerical evidence which we will present in our forthcoming article [6]. 
Conjecture 3.5 Let $\beta>1$. Then there is a value $t_{\beta} \in(0, \pi / 2)$ such that for each $t \in\left(0, t_{\beta}\right)$ there are exactly two values of $\gamma$ such that $M(G(\beta, \gamma))=$ $M(t)$. (For $t=t_{\beta}$ there is exactly one, while for $t>t_{\beta}$ there are none.)

We now have in hand enough information about gears to prove one of our main results.

Theorem 3.6 For each $t$ in $(0, \pi / 2)$ there are constants $\lambda_{t}^{-}, \lambda_{t}^{+}$such that $R_{t, \lambda}$ is the Schwarzian derivative of a conformal mapping from $\mathbb{D}$ to a gear domain if and only if $\lambda_{t}^{-}<\lambda<\lambda_{t}^{+}$.

Proof. By Proposition 3.3 there exist $\beta_{0}, \gamma_{0}$ such that $M\left(G_{\beta_{0}, \gamma_{0}}\right)=M(t)$. By the Riemann Mapping Theorem and Theorem 2.2, there exists $\lambda_{0} \in \mathbb{R}$ such that $R_{t, \lambda_{0}}$ is the Schwarzian derivative of a conformal mapping from $\mathbb{D}$ to $G_{\beta_{0}, \gamma_{0}}$. Since the set of $\lambda \in \mathbb{R}$ for which $R_{t, \lambda}$ is the Schwarzian derivative of a conformal mapping from $\mathbb{D}$ to a gear domain is clearly open, we may consider the maximal open interval $I_{t}=\left(\lambda_{t}^{-}, \lambda_{t}^{+}\right)$which contains $\lambda_{0}$ and is contained in this set. Suppose that there is a sequence $\lambda_{n} \rightarrow \lambda_{t}^{+}$in $I_{t}$ for which the gear ratio $\gamma_{n}$ converges to some $\gamma_{0} \in(0, \pi)$. The corresponding conformal mappings $f_{n}: \mathbb{D} \rightarrow G_{\beta_{n}, \gamma_{n}}$ with $\mathcal{S}_{f_{n}}=R_{t, \lambda_{n}}$, symmetric in $\mathbb{R}$, and with $f_{n}^{\prime}(0)>0$, all cover $\mathbb{D}$ and thus (perhaps on a subsequence) converge to a mapping $f$ with $\mathbb{D} \subseteq f(\mathbb{D})$. Since $\lambda_{t}^{+}$is a boundary parameter, we must have $\beta_{n} \rightarrow 1$ or $\beta_{n} \rightarrow \infty$ on a subsequence. However, by Proposition 3.3 this implies $M\left(G_{\beta_{n}, \gamma_{n}}\right) \rightarrow 0$ or $M\left(G_{\beta_{n}, \gamma_{n}}\right) \rightarrow \infty$, which is absurd since the conformal module $M(t)$ is fixed. It follows that $\gamma$ accumulates only to 0 or $\pi$ as $\lambda \rightarrow \lambda_{t}^{+}$, and the same holds as $\lambda \rightarrow \lambda_{t}^{-}$.

However, the function $\lambda \mapsto \gamma$ (for the fixed $t$ under consideration) is strictly monotone. Indeed, suppose that the Schwarzian derivatives $R_{t, \lambda_{1}}$ and $R_{t, \lambda_{2}}$ determined gears $G_{\beta_{1}, \gamma}$ and $G_{\beta_{2}, \gamma}$ with the same $\gamma$ but $\beta_{1}<\beta_{2}$. Then $G_{\beta_{1}, \gamma} \subseteq G_{\beta_{2}, \gamma}$ and by monotonicity of conformal modules, $M\left(G_{\beta_{2}, \gamma}\right)<$ $M\left(G_{\beta_{1}, \gamma}\right)$, again contradicting the fact that both modules are equal to $M(t)$. We thus see that $\gamma \rightarrow \pi$ as $\lambda \rightarrow \lambda_{t}^{-}$and $\gamma \rightarrow 0$ as $\lambda \rightarrow \lambda_{t}^{+}$, or vice versa. (Numerically we will see in [6] that $\lambda \mapsto \gamma$ is actually decreasing.)

Now we can show that $R_{t, \lambda}$ is not the Schwarzian derivative of a gear mapping for any $\lambda$ outside of the interval $I_{t}$. Suppose indeed that $\lambda_{1}$ were such a parameter. As was the case for $\lambda_{0}$, there is a maximal interval $I$ containing $\lambda_{1}$ and in which every $R_{t, \lambda}$ gives a gear mapping. As before, the 
limits of the $\gamma$ values at the endpoints of $I$ must be 0 and $\pi$, and these values thus range over the whole interval $(0, \pi)$. Therefore there exists a value $\lambda \in I$ such that $R_{t, \lambda}$ produces a gear $G_{\beta, \gamma_{0}}$, where $R_{t, \lambda_{0}}$ produces $G_{\beta_{0}, \gamma_{0}}$. Again by monotonicity of the module we have that $\beta=\beta_{0}$. Then by the uniqueness of conformal mappings, $R_{t, \lambda}=R_{t, \lambda_{0}}$ and finally $\lambda=\lambda_{0}$. This proves the statement.

(The limiting rational expressions $R_{t, \lambda_{t}^{ \pm}}$correspond to maps to degenerate gears, as will be discussed now).

\section{The region of gearlikeness}

\subsection{Pregear domains}

Definition 4.1 We will say that a domain $D$ is a pregear when it is the image $D=T(G)$ of a one-tooth gear domain $G$ under a Möbius transformation $T$.

The tooth edges as well as the A- and B-arcs of any pregear may be uniquely identified by the corresponding interior angles. Normally we will restrict the discussion to pregears with the symmetries of the following proposition.

Proposition 4.2 Let $D$ be a circular quadrilateral which is symmetric in $\mathbb{R}$, has no vertices on $\mathbb{R}$, and has two interior angles equal to $\pi / 2$ and two interior angles equal to $3 \pi / 2$. Assume that one tooth edge of $D$ lies in the upper and the other in the lower half-plane. Then $D$ is a pregear if and only if the full circles $C^{+}, C^{-}$containing the tooth edges intersect in two points.

Proof. Suppose that $C^{+}$and $C^{-}$intersect at the points $b^{-}$and $b^{+}$, which by symmetry are necessarily in $\mathbb{R}$. We can suppose that $b^{-}<b^{+}$. If the A-arc passes between $b^{-}$and $b^{+}$as in diagrams (5) and (6) in Figure 2, then $b^{+}$is interior to $D$. Otherwise, if the B-arc passes between $b^{-}$and $b^{+}$as in diagrams (1), (2), (3) and (4) in Figure 2, then $b^{-}$is interior to $D$. Let $T$ be a Möbius transformation such that $T^{-1}(z)=\left(z-b^{-}\right) /\left(z-b^{+}\right)$. Then $T^{-1}\left(C^{+}\right)$and $T^{-1}\left(C^{-}\right)$are straight lines which pass through the origin, so $D=T(G)$, where $G$ is a gear domain (or the image of a gear domain under $z \mapsto 1 / z)$. For the converse, when the extended tooth edges intersect in 
(1)

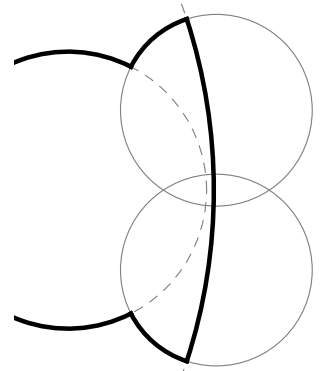

(4)

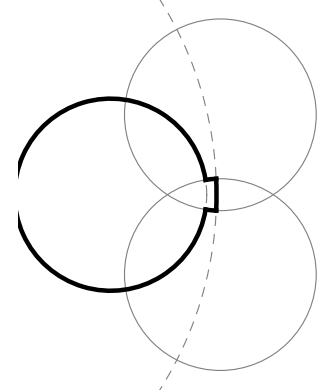

(2)

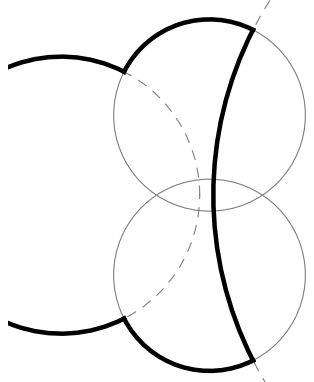

(5)

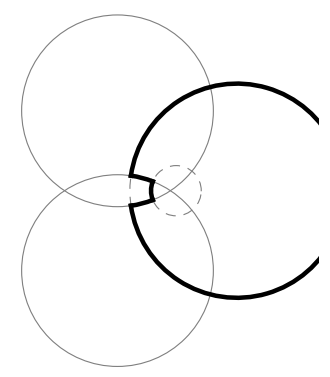

(3)

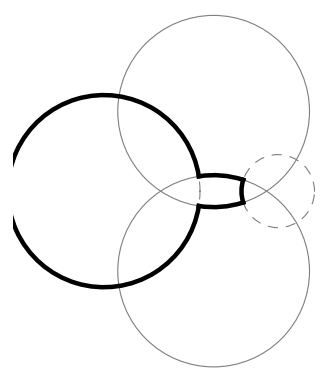

(6)

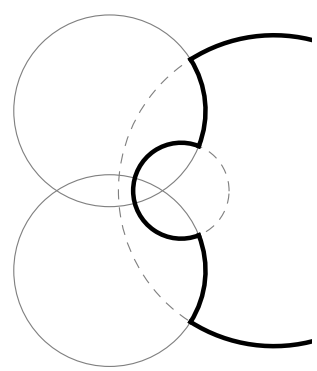

Figure 2: Examples of pregears. The circles $C^{ \pm}$containing the tooth edges are solid gray; the circles containing the A- and B-arcs in dotted gray.

two points, $C^{+} \cap C^{-}=\{T(0), T(\infty)\}$ where $T$ is a Möbius transformation sending some $G_{\beta, \gamma}$ to the pregear $D$.

Proposition 4.3 Let $D$ be a pregear. Then $D$ is a gear if and only if its tooth edges are straight, or equivalently, if the $A$-and $B$-arcs are concentric.

\subsection{Degenerate gears and pregears}

Definition 4.4 A circular quadrilateral is called a degenerate gear (or pregear) if it is not a gear (or pregear) but is arbitrarily close to one.

Naturally a degenerate gear is also a degenerate pregear. Degenerate pregears have a very rigidly defined structure. According to Proposition 4.2, each edge of a pregear is a circular arc orthogonal to the two edges adjacent to it, and the tooth edges lie in circles $C^{ \pm}$which intersect in two points. It follows from 
this that in a degenerate pregear the circles $C^{ \pm}$must be tangent. Further, any circle orthogonal to both of them must pass through the point of tangency. From this one may deduce the following.

Proposition 4.5 Let $D$ be a bounded degenerate pregear symmetric in $\mathbb{R}$. Then the circles $C^{ \pm}$containing the tooth edges are tangent, say at some point $w^{*}$, and are orthogonal to the A- and B-circles (the circles containing the non-tooth edges), which are also tangent at $w^{*}$.
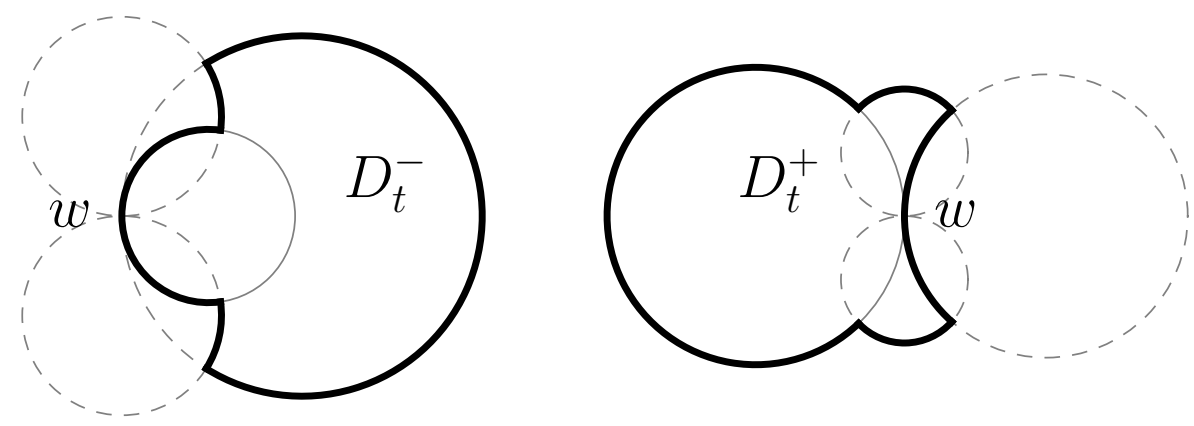

Figure 3: The two types of degenerate pregears

The two essentially different possibilities allowed by Proposition 4.5 are when the point of tangency $w^{*}$ of the tooth edges is at what we have been calling $f(-1)$ or at $f(1)$, i.e., according to whether the circles containing the A- and B-arcs are tangent internally or externally, as shown in Figure 3. We will denote by $D_{t}^{-}$and $D_{t}^{+}$respectively the two degenerate pregears with conformal module $M(t), 0<t<\pi$. For definiteness in the following discussion we normalize these domains as follows. For $D_{t}^{-}$we apply a real Möbius transformation to assure that the A-circle is $\{|w+1 / 2|=1 / 2\}$ and the B-circle is $\{|w|=1\}$, so the point of internal tangency of $C^{ \pm}$is at $w=$ $-1 \in \partial D_{t}^{-}$. This normalization does not affect the conformal module. For $D_{t}^{+}$we will assume that the A- and B-circles are $\{|w|=1\}$ and $\{|w-2|=1\}$ respectively, with external tangency at $w=1 \in \partial D_{t}^{+}$.

Let us note the limiting behavior of degenerate pregears as $t \rightarrow 0$ or $t \rightarrow$ $\pi / 2$. Let $\eta$ denote the common radius of $C^{ \pm}$. Consider first $D_{t}^{-}$. When $\eta \rightarrow 0$ it can be seen that the conformal module $M(t)$ tends to $\infty$ (for example, for each $\eta$ apply a Möbius transformation leaving the A-circle invariant and sending $C^{ \pm}$to circles of radius 1 ). In the other direction, when $\eta \rightarrow \infty$, the 


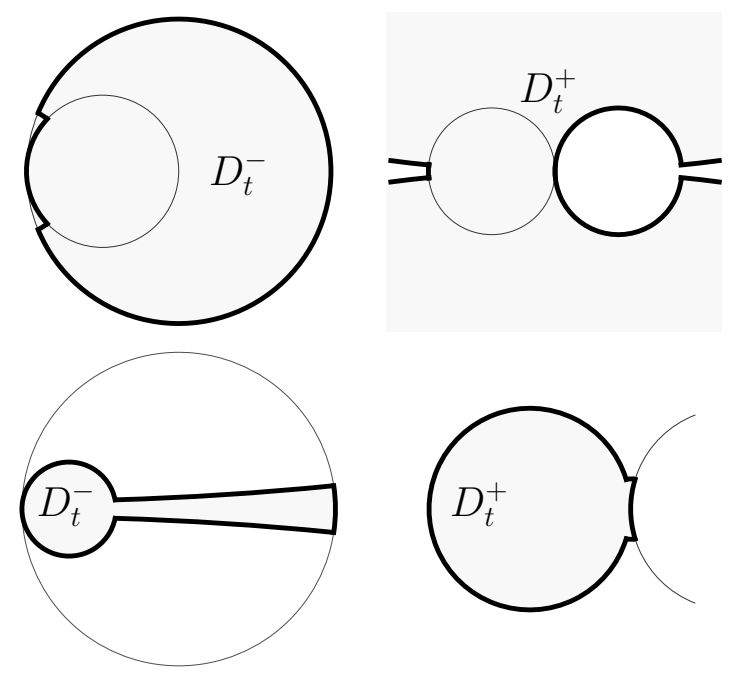

Figure 4: Extreme cases of degenerate pregears, for $\eta \rightarrow 0$ (above) and $\eta \rightarrow \infty$ (below).

A-arc is practically all of the A-circle, and the B-arc is a small arc of the B-circle near 1 , so $M(t) \rightarrow 0$ (see Figure 4). Similarly, for $D_{t}^{+}$, as $\eta \rightarrow 0$ we have $M(t) \rightarrow \infty$, while as $\eta \rightarrow \infty$ we find that $M(t) \rightarrow 0$.

\subsection{The boundary of the region of gearlikeness}

Our main object of study is the the following.

Definition 4.6 The region of gearlikeness is the subset of $\mathbb{R}^{2}$ defined by

$$
\mathcal{G}=\left\{(t, \lambda): R_{t, \lambda} \text { is the Schwarzian derivative of a gear mapping }\right\} .
$$

According to Theorem 3.6,

$$
\mathcal{G}=\left\{(t, \lambda): 0<t<\frac{\pi}{2}, \lambda_{t}^{-}<\lambda<\lambda_{t}^{+}\right\}
$$

One may obtain very rough bounds on $\lambda_{t}^{-}$and $\lambda_{t}^{+}$by the classical estimate of Nehari which says that if $\left(1-|z|^{2}\right)^{2}\left|\mathcal{S}_{f}(z)\right|>6$ for some $z \in \mathbb{D}$, then the mapping $f$ is not even univalent. Applying $z=0$ in (15) gives the necessary condition $\left|16 \lambda \cos t+\sin ^{2} t\right|<6$ for univalence, from which

$$
\lambda_{t}^{-} \geq-\frac{13-\cos 2 t}{32 \cos t}, \quad \lambda_{t}^{+} \leq \frac{11+\cos 2 t}{32 \cos t} .
$$


This approach was worked out in detail for the analogous case of symmetric quadrilaterals in [5], where the Nehari estimate gave rather better results. Indeed we give the following exact values for $\lambda_{t}^{+}, \lambda_{t}^{+}$.

Theorem 4.7 Let $0<t<\pi / 2$. Then the extreme values of $\lambda \in \mathbb{R}$ for which the rational function $R_{t, \lambda}$ of (14) is gearlike are given by

$$
\lambda_{t}^{-}=-\frac{1}{4}-\frac{1}{16}\left(\cos t+\frac{1}{\cos t}\right), \quad \lambda_{t}^{+}=\frac{1}{4}-\frac{1}{16}\left(\cos t+\frac{1}{\cos t}\right) .
$$

Further, $R_{t, \lambda_{t}^{-}}, R_{t, \lambda_{t}^{+}}$are the Schwarzian derivatives of conformal mappings from the disk $\mathbb{D}$ onto degenerate pregears of type $D_{t}^{-}, D_{t}^{+}$respectively, as depicted in Figure 3 .

The region of gearlikeness $\mathcal{G}$ is drawn in Figure 5, together with the rough bound (19).

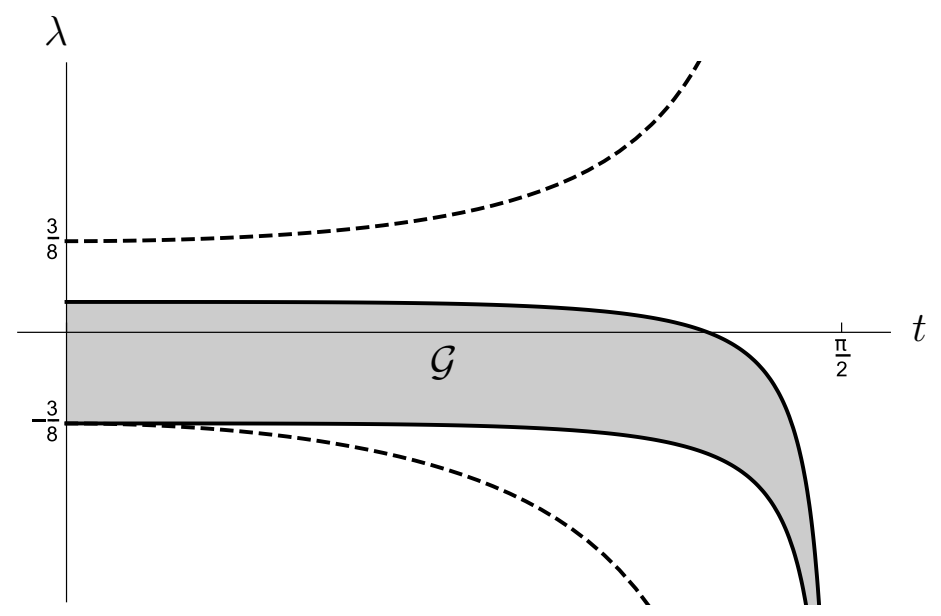

Figure 5: Region of gearlikeness (gray, bounded by solid curves, which correspond to degenerate gears).

Proof. For fixed $t$, as $\lambda \rightarrow \lambda_{t}^{-}$from above and $\lambda \rightarrow \lambda_{t}^{+}$from below, it is difficult to describe immediately the behavior of the parameters of the corresponding gear domains $G_{\beta, \gamma}$. However, via apropriate Möbius transformations we can map these gears to pregears with the A- and B-circles normalized so that the limiting domains are the degenerate pregears $D_{t}^{ \pm}$(recall Proposition 4.5). Then let us apply a real Möbius transformation sending 


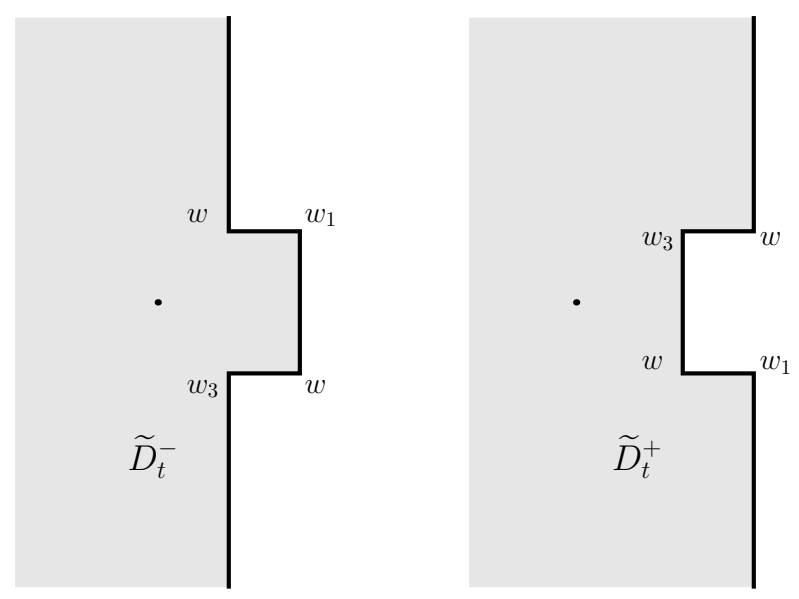

Figure 6: Degenerate pregears with tangency mapped to $\infty$

the point of tangency $w^{*}$ of the extended edges of the pregear to $\infty$. Since all image edges are now straight, the result $\widetilde{D}_{t}^{ \pm}$is an unbounded rectilinear quadrilateral of a very simple form (Figure 6 ). The limit mappings $\mathbb{D} \rightarrow \widetilde{D}_{t}^{ \pm}$ are given by integrals of Schwarz-Christoffel type with prevertices at $\pm e^{ \pm i t}$, and also at -1 or 1 which are mapped to vertices of angle $\pi$ at $\infty$ (cf. [7, Sec. 2.1] for an explanation of this technical detail):

$$
\begin{aligned}
& \tilde{f}_{t}^{-}(z)=\int_{0}^{z} \frac{1}{(z+1)^{2}} \sqrt{\frac{z^{2}+(2 \cos t) z+1}{z^{2}-(2 \cos t) z+1}} d z \\
& \tilde{f}_{t}^{+}(z)=\int_{0}^{z} \frac{1}{(z-1)^{2}} \sqrt{\frac{z^{2}+(2 \cos t) z+1}{z^{2}-(2 \cos t) z+1}} d z .
\end{aligned}
$$

From this the Schwarzian derivatives of the pregear mappings $f_{t}^{ \pm}(z): \mathbb{D} \rightarrow D_{t}^{ \pm}$ are equal to those of the Schwarz-Christoffel integrals $\widetilde{f}_{t}^{ \pm}(z)$. These are very easily calculated, and in the notation of (15)-(16) we have

$$
\begin{aligned}
& \mathcal{S}_{\widetilde{f}_{t}^{-}}(z)=-\frac{3+8 \cos t+\cos 2 t}{2\left(z^{4}-(2 \cos 2 t) z^{2}+1\right)}+\psi_{0, t}(z), \\
& \mathcal{S}_{\widetilde{f}_{t}^{+}}(z)=-\frac{3-8 \cos t+\cos 2 t}{2\left(z^{4}-(2 \cos 2 t) z^{2}+1\right)}+\psi_{0, t}(z)
\end{aligned}
$$

Upon comparison with

$$
R_{t, \lambda}(z)=\frac{16 \lambda \cos t}{z^{4}-(2 \cos 2 t) z^{2}+1}+\psi_{0, t}(z)
$$


we obtain the formulas for $\lambda_{t}^{-}$and $\lambda_{t}^{+}$.

\section{Discussion and conclusions}

We have described in detail the structure of gear and pregear domains and shown how the general theory of conformal mapping to circular polygons can be used to relate the geometry of these domains to the auxiliary parameters of the corresponding conformal mappings. In particular, the conformal module $M(t)$ is a key element for understanding the degeneration of these domains.

In order to calculate a gear domain numerically by the approach we have presented here, given a pair of parameters $(t, \lambda)$ it is necessary to find the appropriate self-mapping $T_{q}$ of (10). This amounts to knowing the point $q \in[-1,1]$ which is sent to the gear center. Since solving the Schwarzian differential equation (18) a priori produces only a pregear, it is necessary to find a Möbius transformation which maps this to a gear. This may be approached by solving numerically for the intersection points of $C^{+} \cap C^{-}$ of the circles containing the tooth edges, or by finding the curvature of the tooth edges numerically and then adjusting the parameters to assure that this curvature is zero. Once all this is obtained, the matter of how to find the unique $(t, \lambda)$ corresponding to prescribed geometric parameters $(\beta, \gamma)$ can be addressed. These questions will worked out in the study [6] of numerical aspects of conformal mappings to gear domains.

\section{References}

[1] R. W. Barnard, K. Pearce, "Rounding corners of gearlike domains and the omitted area problem," J. Comput. Appl. Math. 14:1-2, Special issue on numerical conformal mapping (1986) 217-226 MR0829040.

[2] P. Bjørstad, E. Eric Grosse, "Conformal mapping of circular arc polygons," SIAM J. Sci. Statist. Comput. 8 (1987) 19-32 MR0873921.

[3] P. Brown, "An investigation of a two parameter problem for conformal maps onto circular arc quadrilaterals," Complex Var. Elliptic Equ. 53 no. 1 (2008) 23-51 MR2380819. 
[4] P. Brown, "Conformal mapping of a gear domain with one tooth," Quaest. Math. 33:3 (2010) 277-289 MR2755521.

[5] P. Brown, R. M. Porter, "Conformal mapping of circular quadrilaterals and Weierstrass elliptic functions," Comput. Methods Funct. Theory 11:2 (2011) 463-486 MR2858958.

[6] P. Brown, R. M. Porter, "Computation of conformal mappings to gearshaped planar domains" (submitted).

[7] T. A. Driscoll, L. N. Trefethen, Schwarz-Christoffel Mapping, Cambridge Monogr. Appl. Comput. Math., Cambridge University Press, Cambridge (2002) MR1908657.

[8] A. W. Goodman, "Conformal mapping onto certain curvilinear polygons," Univ. Nac. Tucumn Rev. Ser. A 13 (1960) 20-26 MR0146364.

[9] E. Hille, Analytic Function Theory, Vol. 2, Introductions to Higher Mathematics, Ginn and Co., Boston, Mass.-New York-Toronto, Ont. (1962) MR0201608.

[10] V. V. Kravchenko and R. M. Porter, "Conformal Mapping of Right Circular Quadrilaterals," Complex Var. Elliptic Equ. 56:5 (2011) 399415 MR2795464.

[11] O. Lehto and K. I. Virtanen, Quasiconformal Mappings in the Plane, second edition, Die Grundlehren der mathematischen Wissenschaften 126 Springer-Verlag, New York-Heidelberg (1973).

[12] Z. Nehari, Conformal Mapping, McGraw-Hill Book Co., New YorkToronto-London (1952) MR0377031.

[13] H. Nishimiya, "Conformal mapping onto gearlike domain," Kodai Math. Sem. Rep. 16:4 (1964) 243-248 MR0182719.

[14] K. Pearce, "A constructive method for numerically computing conformal mappings for gearlike domains," SIAM J. Sci. Statist. Comput. 12:2 (1991) 231-246 MR1087758

[15] R. M. Porter, "Numerical calculation of conformal mapping to a disk minus finitely many horocycles," Comput. Methods Funct. Theory 5:2 (2005) 471-488 MR2205427. 
Philip R. Brown

Department of General Academics

Texas A\&M University at Galveston

PO Box 1675, Galveston, Texas 77553 -1675

brownp@tamug.edu

R. Michael Porter

Departamento de Matemáticas, CINVESTAV-I.P.N.

Apdo. Postal 1-798, Arteaga 5

Santiago de Queretaro, Qro., 76000 MEXICO

mike@math. cinvestav.edu.mx 